Математички Билтен

ISSN 0351-336X (print)

43 (LXIX) No.1

ISSN 1857-9914 (online)

$2019(27-46)$

UDC: $514.144: 512.714$

Скопје, Македонија

\title{
THE CONSTRUCTION OF A CORP IN THE SET OF POINTS IN A LINE OF DESARGUES AFFINE PLANE
}

\author{
KRISTAQ FILIPI, ORGEST ZAKA, AND AZIR JUSUFI
}

\begin{abstract}
In the article 1], we show that the set of points on a line, in the affine Desargues plans, connected with addition forms an Abelian group. In this article, we will define multiplication of points on a line in the affine Desargues plans. We will show that this set forms a multiplicative group. And we will show that every straight line of Desargues affine plans, along with both addition and multiplication operations, forms the corp (skew-field).
\end{abstract}

\section{Introduction, Desargues affine plane, COMMUTATIVE GROUP $(O I,+)$}

Definition 1. [3, 10, 11] Affine plane is called the incidence structure $\mathcal{A}=$ $(\mathcal{P}, \mathcal{L}, \mathcal{I})$ that satisfies the following axioms:

Axiom 1. For every two different points $P$ and $Q \in \mathcal{P}$, there exists exactly one line $\ell \in \mathcal{L}$ incident with that points.

The line $\ell$, determined from the point $P$ and $Q$ will be denoted by $P Q$.

Axiom 2. For a point $P \in \mathcal{P}$, and an line $\ell \in \mathcal{L}$ such that $(P, \ell) \notin \mathcal{I}$, there exists one and only one line $r \in \mathcal{L}$ incident with the point $P$ and such that $\ell \cap r=\varnothing$.

Axiom 3. In $\mathcal{A}$ there are three non-incident points with a line.

2010 Mathematics Subject Classification. 51-XX, 51Axx, 51Exx, 51E15, 12Exx, $12 \mathrm{E} 15$.

Key words and phrases. Affine Desargues plane, additions of point, multiplication of points, subgroup, group, Abelian group, skew-field (corp). 


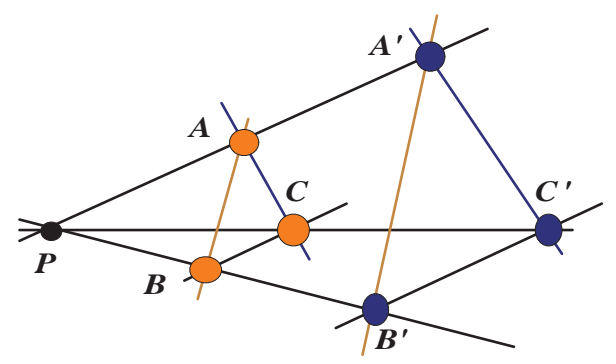

FIGURE 1.

The fact $(P, \ell) \in \mathcal{I}$, (equivalent to $P \mathcal{I} \ell$ ) we mark $P \in \ell$ and read point $P$ is incident with a line $\ell$ or a line passes through points $P$ (contains point $P)$. Whereas a line of the affine plane we consider as sets of points of affine plane with her incidents. Axiom 1 implicates that tow different lines of $\mathcal{L}$ many have a common point, in other words tow different lines of $\mathcal{L}$ either have no common point or have only one common point.

Definition 2. Two lines $\ell, m \in \mathcal{L}$ that are matching or do not have common point are called parallel and in this case we write $\ell \| m$; when they have only one common point we say that they are expected.

For a single line $r \in \mathcal{L}$, which passes through a point $P \in \mathcal{P}$ and is parallel with line $A B$, that does not pass through the point $P$, we will use the notation $\ell_{A B}^{P}$.

Proposition 1.1. [4, 10, 12, 13] Parallelism relation $\|=\left\{(r, s) \in \mathcal{L}^{2} \mid r \| s\right\}$ on $\mathcal{L}$ is an equivalence relation in $\mathcal{L}$.

Definition 3. Three different points $P, Q, R \in \mathcal{P}$ are called collinear, if there is incidence with the same straight line.

Definition 4. The set of three different non-collinear points $A, B, C$ together with the line $A B, B C, C A$ is called three-vertex and is marked as $A B C$.

Proposition 1.2. 6, 7, 9, 10, 22] (The Desargues affine plane theorem). If $A B C, A^{\prime} B^{\prime} C^{\prime}$ are two three-vertex but not with the same vertices in an affine plane (Fig. 1), then

$$
\begin{aligned}
& A C \| A^{\prime} C^{\prime} \\
& B C \| B^{\prime} C^{\prime}
\end{aligned} \Longrightarrow A B \| A^{\prime} B^{\prime}
$$

In affine Euclidean plane this proposition holds 


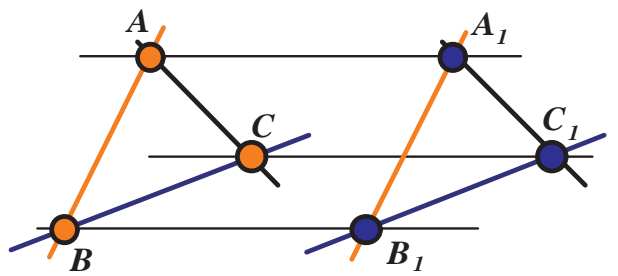

FiguRE 2.

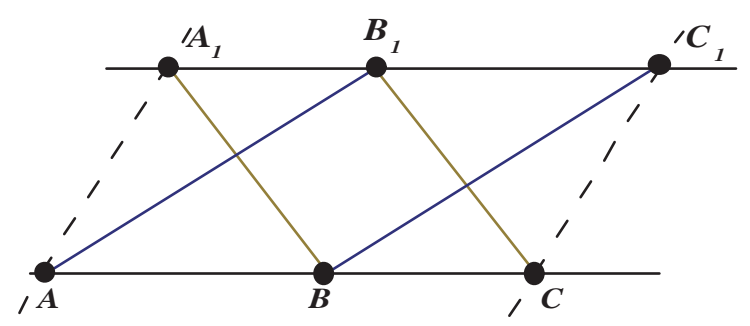

FiguRE 3.

Proposition 1.3. (Axiom I of Desargues) If $A A_{1}, B B_{1}, C C_{1}$ are three different parallel lines (Fig. 2), then

$$
\begin{aligned}
& A B \| A_{1} B_{1} \\
& B C \| B_{1} C_{1}
\end{aligned} \Longrightarrow A C \| A_{1} C_{1}
$$

There are affine plans where Proposition 1.3 is not valid. Such is the Moulton plane [10].

Definition 5. [2, 7, 10] An affine plane complete with Desargues axiom (Proposition 1.3), is called Desargues affine plane.

Let $A, B, C$ be three different points of a line and $A_{1}, B_{1}, C_{1}$ three different points of another parallel to the first (Fig.3). If $A B_{1} \| B C_{1}$ and $A_{1} B \| B_{1} C$ is $A A_{1} \| C C_{1}$ ? Otherwise, we add the problem if we have this

Proposition 1.4. [1, 17, 18] ("Little Pappus Theorem"). Let A, B, C and $A_{1}, B_{1}, C_{1}$ be two triple points located in two parallel lines (Fig. 3). If $A B_{1} \| B C_{1}$ and $B A_{1} \| C B_{1}$ then $A A_{1} \| C C_{1}$ holds.

Theorem 1. [1, 17] ("Little Hessenberg Theorem") For a Desargues plane Propositions 1.4 is true. 


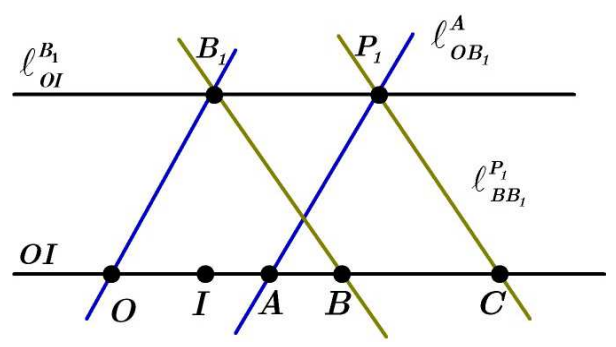

FigURE 4.

In an Desargues affine plane $\mathcal{D}=(\mathcal{P}, \mathcal{L}, \mathcal{I})$ we fix two different points $O, I \in \mathcal{P}$, which, according to Axiom 1 , determine a line $O I \in \mathcal{L}$. Let $A$ and $B$ be two arbitrary points of a line $O I$. In plane $\mathcal{D}$ we choose a point $B_{1}$ not incident with $O I: B_{1} \notin O I$ (we call the auxiliary point). Construct line $\ell_{O I}^{B_{1}}$, which is only according to the Axiom 2. Then construct line $\ell_{O B_{1}}^{A}$, which also is the only according to the Axiom 2. Marking their intersection $P_{1}=\ell_{O I}^{B_{1}} \cap \ell_{O B_{1}}^{A}$. Finally construct line $\ell_{B B_{1}}^{P_{1}}$. For as much as $B B_{1}$ expects $O I$ in point $B$, then this line, parallel with $B B_{1}$, expects line $O I$ in a single point $C$ (Fig.4).

The process of construct the points $C$, starting from two whatsoever points $A, B$ of the line $O I$, is presented in the algorithm form

\section{Algorithm 1.}

$$
\begin{aligned}
& \text { Step.1. } B_{1} \notin O I \\
& \text { Step.2. } \ell_{O I}^{B_{1}} \cap \ell_{O B_{1}}^{A}=P_{1} \\
& \text { Step.3. } \ell_{B B_{1}}^{P_{1}} \cap O I=C
\end{aligned}
$$

The point $C$ is determined in single mode (does not depend on the choice auxiliary point $B_{1}$ ) by Algorithm 1 [1] .

Definition 6. [1] In the above conditions, operation

$$
+: O I \times O I \longrightarrow O I
$$

defined by $(A, B) \mapsto C$ for all $(A, B) \in O I \times O I$ we call the addition in $O I$.

According to this definition, one can write

Step.1. $B_{1} \notin O I$

$(\forall A, B \in O I)$ Step.2. $\ell_{O I}^{B_{1}} \cap \ell_{O B_{1}}^{A}=P_{1} \quad \Leftrightarrow A+B=C$.

Step.3. $\ell_{B B_{1}}^{P_{1}} \cap O I=C$ 


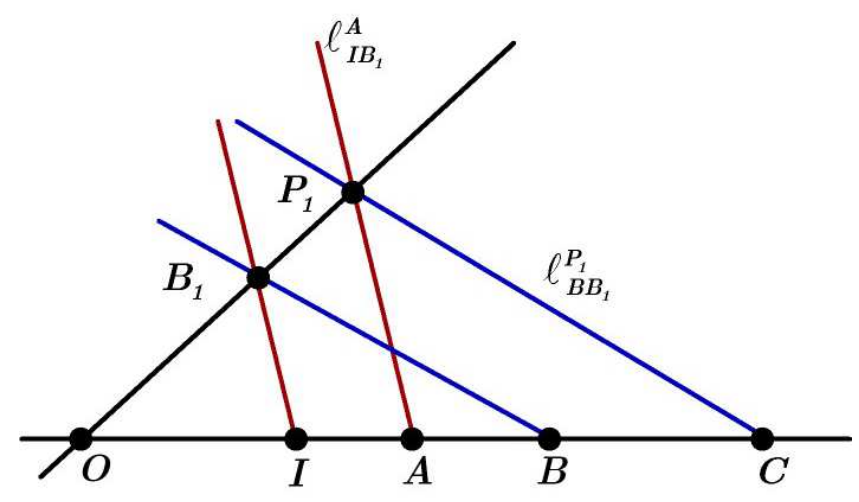

FiguRE 5.

Theorem 2. [1] The groupoid $(O I,+)$ is commutative (Abelian) group; the zero element is the point $O$.

\section{Multiplication of points on a line in Desargues affine PLANE AND ITS PROPERTIES}

Choose in the plane $\mathcal{D}$ one point $B_{1}$ not incident with lines $O I$, which together with point $I$ forming the line $I B_{1}$. Construct the line $\ell_{I B_{1}}^{A}$, which is the only according to the Axiom 2 and cutting the line $O B_{1}$. Marking their intersection with $P_{1}=\ell_{I B_{1}}^{A} \cap O B_{1}$. Finally, construct the line $\ell_{B B_{1}}^{P_{1}}$. Since $B B_{1}$ meets the line $O I$ in point $B$, then this line, parallel with $B B_{1}$, meets the line $O I$ in one single point $C$ (Fig.5).

The process of construct the points $C$, is presented in the algorithm form

\section{Algorithm 2.}

$$
\begin{array}{ll}
\text { Step.1. } & B_{1} \notin O I \\
\text { Step.2. } & \ell_{I B_{1}}^{A} \cap O B_{1}=P_{1} \\
\text { Step.3. } & \ell_{B B_{1}}^{P_{1}} \cap O I=C
\end{array}
$$

In the process of construct the points $C$, except pairs $(A, B)$ of points $A, B \in O I$, is required and the selection of point $B_{1} \notin O I$, which we call the auxiliary point to point $C$. The following theorem demonstrates that the choice of auxiliary point does not affect the position of point $C$ in line $O I$, determined by the Algorithm 2 . 


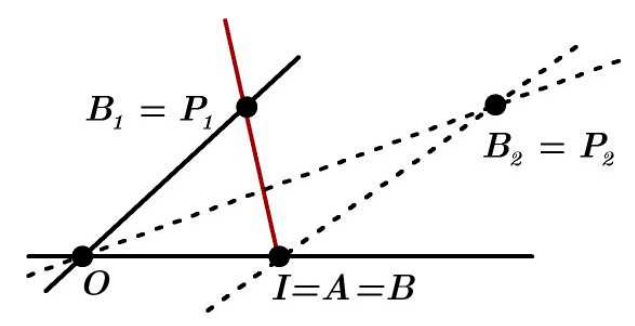

Figure 6.

Theorem 3. For every two points $A, B \in O I$, the Algorithm 2 determines a single point $C \in O I$, which does not depend on the choice of its auxiliary point $B_{1}$.

Proof. According to the Algorithm 2, by selecting the point $B_{1} \notin O I$ for a given pair of points $(A, B)$ of the line $O I$, construct the point $C$. Now we choose another point $B_{2}$. Then, according to Algorithm 2, construct analog the point $C^{\prime}$, that in these conditions is found as:

$$
\left[\begin{array}{l}
\text { Step.1. } B_{2} \notin O I \\
\text { Step.2. } \ell_{O I}^{B_{2}} \cap \ell_{O B_{2}}^{A}=P_{2} \\
\text { Step.3. } \ell_{B B_{2}}^{P_{2}} \cap O I=C^{\prime}
\end{array}\right]
$$

We distinguish these four cases of the position of points $A, B$ in relation to fixed point $I$ of the line $O I$.

Case 1. $A=B=I$. By the choice of the point $B_{1}$, according to Algorithm 2, have:

$$
P_{1}=\ell_{I B_{1}}^{I} \cap O B_{1}=B_{1} \Longrightarrow C=\ell_{B B_{1}}^{B_{1}} \cap O I=I B_{1} \cap O I=I
$$

From the choice of the point $B_{2}$, according to 2.1 have:

$$
P_{2}=\ell_{I B_{2}}^{I} \cap O B_{2}=B_{2} \Longrightarrow C^{\prime}=\ell_{I B_{2}}^{B_{1}} \cap O I=I B_{2} \cap O I=I .
$$

Therefore accept the $C=C^{\prime}=I$ (Fig.6).

Case 2. $A=I \neq B$. By the choice of the point $B_{1}$ have

$$
P_{1}=\ell_{I B_{1}}^{I} \cap O B_{1}=B_{1} \Longrightarrow C=\ell_{B B_{1}}^{B_{1}} \cap O I=B B_{1} \cap O I=B ;
$$

From the choice of the point $B_{2}$ have

$$
P_{2}=\ell_{I B_{2}}^{I} \cap O B_{2}=B_{2} \Longrightarrow C=\ell_{B B_{2}}^{B_{2}} \cap O I=B B_{2} \cap O I=B .
$$

Therefore in this case accept the $C=C^{\prime}=B$ (Fig.7). 
CORP IN THE SET OF POINTS IN A LINE OF DESARGUES AFFINE PLANE 33

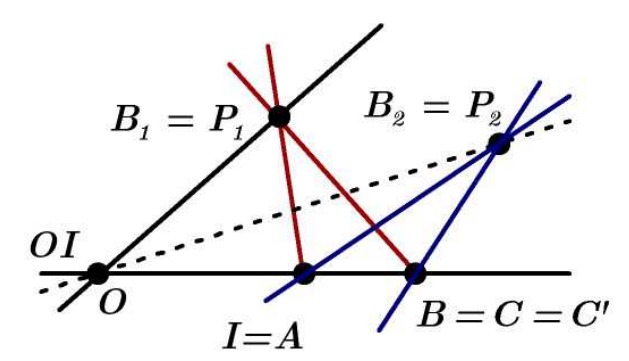

FigURE 7.

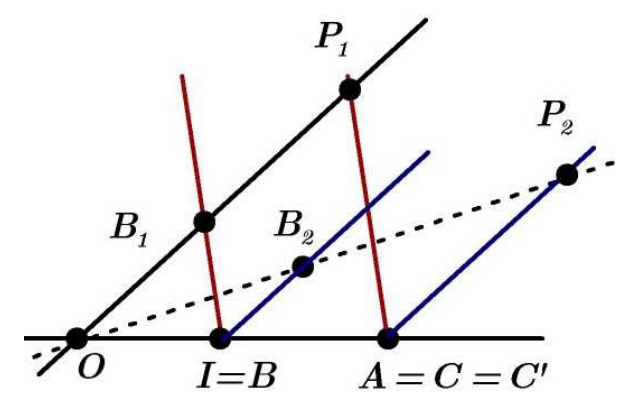

FiguRE 8.

Case 3. $A \neq I=B$. The situation is analogous to the second case, where point $B$ takes the role of point $A$ and conversely, so in this case we have $C=C^{\prime}=A$ (Fig.8).

Case 4. $A \neq B \neq I$. Here we distinguish two sub-cases.

a) In the case where points $I, B_{1}, B_{2}$ are collinear points, by the choice of the point $B_{1}$ have

$$
P_{1}=\ell_{I B_{1}}^{A} \cap O B_{1} \Longrightarrow C=\ell_{B B_{1}}^{P_{1}} \cap O I
$$

from the choice of the point $B_{2}$ have

$$
P_{2}=\ell_{I B_{2}}^{A} \cap O B_{2} \Longrightarrow C^{\prime}=\ell_{B B_{2}}^{P_{2}} \cap O I \text {. }
$$

From Algorithm 2 and (2.1) appears also that, collinearity of points $I, B_{1}, B_{2}$ induce collinearity of the points $A, P_{1}, P_{2}$.

Examine three-vertices $B B_{1} B_{2}$ and $C P_{1} P_{2}$ (Fig.9). We note that $B_{1} B_{2} \| P_{1} P_{2}$. But $C \in \ell_{B B_{1}}^{P_{1}} \| B B_{1}$ therefore $B B_{1} \| C P_{1}$. 


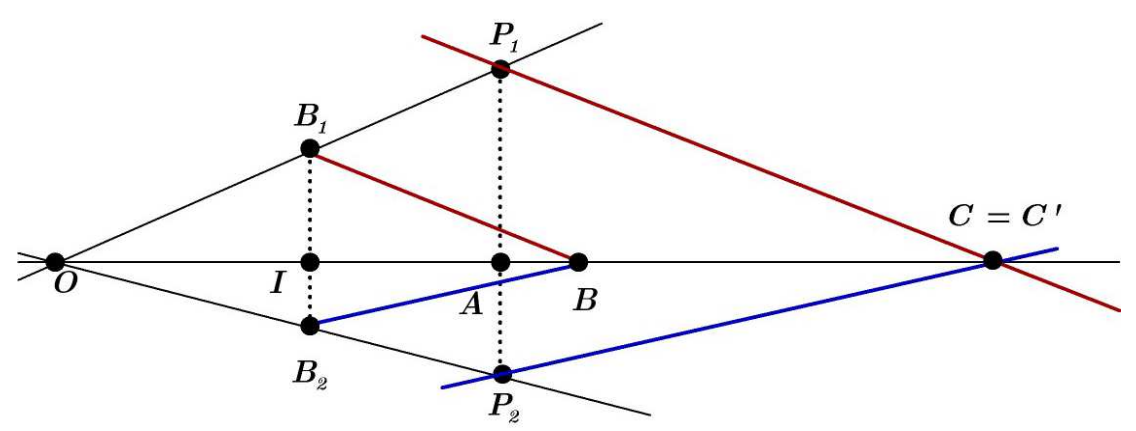

Figure 9.

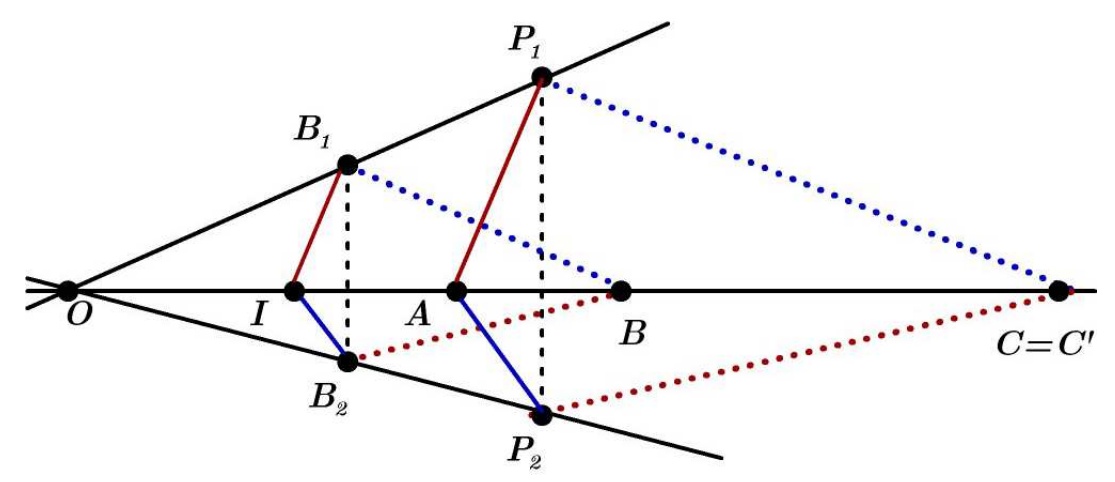

FigURE 10.

From here, the Desargues affine plane theorem (Proposition 1.2), results that $B_{2} B \| P_{2} C$. On the other hand, $C^{\prime} \in \ell_{B B_{2}}^{P_{2}} \Longrightarrow P_{2} C^{\prime} \| B_{2} B$, which is parallel to $P_{2} C$. Consequently $C^{\prime} \in P_{2} C$, which means that $C=C^{\prime}$.

b) The points $I, B_{1}, B_{2}$ are non-collinear. Here we distinguish two subcases related to fixed point $O$ :

$\mathbf{b}_{1}$ ) The points $O, B_{1}, B_{2}$ are non-collinear (Figure 10);

$\mathbf{b}_{2}$ ) The points $O, B_{1}, B_{2}$ are collinear (Figure 11).

In case $\left.\mathbf{b}_{\mathbf{1}}\right)$, from the choice of point $B_{1}$, have: $P_{1}=\ell_{I B_{1}}^{A} \cap O B_{1} \Longrightarrow$ $C=\ell_{B B_{1}}^{P_{1}} \cap O I$

from the choice of point $B_{2}$, according to 2.1 have: $P_{2}=\ell_{I B_{2}}^{A} \cap O B_{2} \Longrightarrow$ $C^{\prime}=\ell_{B B_{2}}^{P_{2}} \cap O I$.

From Algorithm 2, and 2.1) we get also that, non-collinearity of points $I, B_{1}, B_{2}$ delivers non-collinearity of points $A, P_{1}, P_{2}$. 


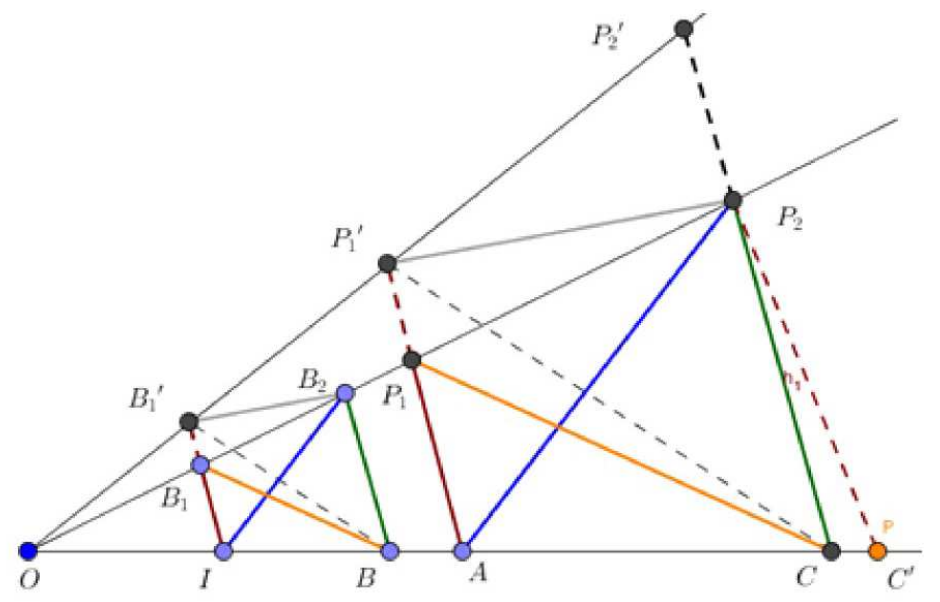

FiguRE 11.

We consider three-vertices $I B_{1} B_{2}$ and $A P_{1} P_{2}$. By Desargues affine plane theorem, we obtain that $B_{1} B_{2} \| P_{1} P_{2}$.

Now consider three-vertices $B B_{1} B_{2}$ and $C P_{1} P_{2}$. Again by Desargues affine plane theorem, we take $B_{2} B \| P_{2} C$. On the other hand, $C^{\prime}$, that delivers $P_{2} C^{\prime} \| B_{2} B$. Consequently $C^{\prime} \in P_{2} C$, which means that $C=C^{\prime}$.

In the case $\mathbf{b}_{\mathbf{2}}$ ), again we have

$P_{1}=\ell_{I B_{1}}^{A} \cap O B_{1} \Longrightarrow C=\ell_{B B_{1}}^{P_{1}} \cap O I ; P_{2}=\ell_{I B_{2}}^{A} \cap O B_{2} \Longrightarrow C^{\prime}=\ell_{B B_{2}}^{P_{2}} \cap O I$.

In the line $C P_{2}$ we take another point $P_{2}^{\prime}$ and construct the line $O P_{2}^{\prime}$. Mark $B_{1}^{\prime}=O P_{2}^{\prime} \cap I B_{1}$ and $P_{1}^{\prime}=O P_{2}^{\prime} \cap A P_{1}$. We examine three-vertices $I B_{1}^{\prime} B_{2}$ and $A P_{1}^{\prime} P_{2}$. We have: $I B_{1}^{\prime}\left\|A P_{1}^{\prime}, I B_{2}\right\| A P_{2}$, therefore, by Desargues affine plane theorem, we take from $B_{1}^{\prime} B_{2} \| P_{1}^{\prime} P_{2}$. Now examine threevertices $B B_{1} B_{1}^{\prime}$ and $C P_{1} P_{1}^{\prime}$; by Desargues affine plane theorem, we take from $B B_{1}^{\prime} \| C P_{1}^{\prime}$. Finally we examine three-vertices $B B_{1}^{\prime} B_{2}$ and $C P_{1}^{\prime} P_{2}$. We have: $B B_{1}^{\prime}\left\|C P_{1}^{\prime}, B_{1}^{\prime} B_{2}\right\| P_{1}^{\prime} P_{2}$, therefore we take from $B B_{2} \| C P_{2}$. But $B B_{2} \| C / P_{2}$, and therefore $C=C^{\prime}$.

Let $A$ and $B$ be two arbitrary points of the line $O I$. We associate pairs $(A, B) \in O I \times O I$ point $C \in O I$, that determine algorithm Algorithm 2.

According to the preceding Theorems, point $C$ is determined in single mode. Thus we obtain an application $O I \times O I \longrightarrow O I$. 
Definition 7. In the above conditions, we call the operation

$$
*: O I \times O I \longrightarrow O I,
$$

defined by $(A, B) \mapsto C$ for all $(A, B) \in O I \times O I$, multiplication in $O I$.

According to this definition, one can write

$$
(\forall A, B \in O I,)\left[\begin{array}{l}
\text { Step1. } B_{1} \notin O I, \\
\text { Step2. } \ell_{I B_{1}}^{A} \cap O B_{1}=P_{1} \\
\text { Step3. } \ell_{B B_{1}}^{P_{1}} \cap O I=C .
\end{array}\right] \Longleftrightarrow A * B=C .
$$

From here, the following proposition is obvious.

$$
(\forall A \in O I) O * A=A * O=O .
$$

\section{Properties of the multiplication in the Line $O I$}

By Theorem 3, this is immediately true.

Proposition 3.1. Multiplication * in OI has identity element the point I:

$$
(\forall A \in O I) I * A=A * I=I .
$$

The following propositions are also valid.

Proposition 3.2. The multiplication $*$ is associative in $O I$ :

$$
(\forall A, B, D \in O I)(A * B) * D=A *(B * D) .
$$

Proof. In the case where at least one of the points $A, B, D$ is point $O$, from (2.3), equation $(3.2)$ is evident, whereas in the case where at least one of the points $A, B, D$ is point $I$, it comes from (3.1). We eliminate in the case where $A, B, D \neq O ; A, B, D \neq I$ and $A \neq B \neq D$ (when at least two points are the same, equally justify).

Firstly we construct the product $(A * B) * D$. In this case (Fig.12), according to 2.2 , for $A * B$, connected to auxiliary point for multiplication we have

$$
\begin{gathered}
\left\{\begin{array}{l}
\text { 1. } B_{1} \notin O I, \\
\text { 2. } \ell_{I B_{1}}^{A} \cap O B_{1}=P_{1}, \\
\text { 3. } \ell_{B B_{1}}^{P_{1}} \cap O I=C .
\end{array}\right\} \Longrightarrow A * B=\ell_{B B_{1}}^{P_{1}} \cap O I \\
\Longrightarrow\left\{\begin{array}{c}
I B_{1} \| A P_{1} \\
B B_{1} \|(A * B) P_{1}
\end{array}\right\}
\end{gathered}
$$


CORP IN THE SET OF POINTS IN A LINE OF DESARGUES AFFINE PLANE 37

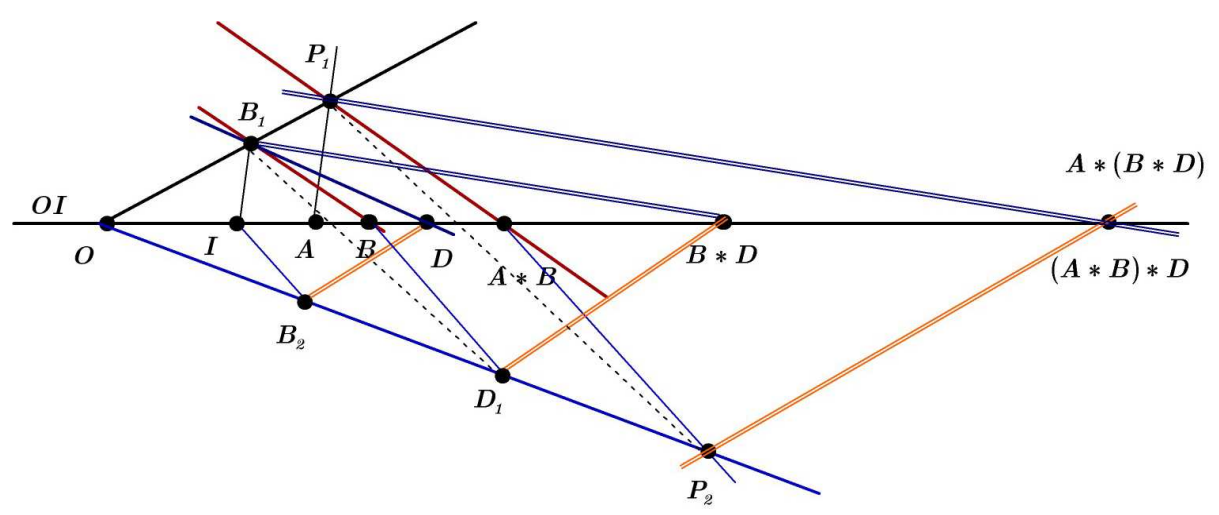

FIGURE 12.

Choose the point $B_{2}$ (Fig.12) as auxiliary points for the construction of multiplication $(A * B) * D$.

Construct the line $\ell_{I B_{2}}^{A * B}$ and mark $P_{2}=\ell_{I B_{2}}^{A * B} \cap O B_{2}$. Then, according to (2.2) have

$$
\begin{aligned}
& \left\{\begin{array}{l}
\text { 1. } B_{2} \notin O I, \\
\text { 2. } O B_{2} \cap \ell_{I B_{2}}^{A * B}=P_{2}, \\
\text { 3. } \ell_{D B_{2}}^{P_{2}} \cap O I=C .
\end{array}\right\} \Longrightarrow(A * B) * D=\ell_{D B_{2}}^{P_{2}} \cap O I \\
& \\
& \Longrightarrow\left\{\begin{array}{c}
I B_{2} \|(A * B) P_{2} \\
D B_{2} \|[(A * B) * D] P_{2}
\end{array}\right\}
\end{aligned}
$$

Now construct multiplication $A *(B * D)$. Choose as the auxiliary point for multiplication $B * D$ the point $B_{2}$. Construct the line $\ell_{I B_{2}}^{B}$ and mark $D_{1}=\ell_{I B_{2}}^{B} \cap O B_{2}$. Then, according to 2.2 have

$$
B * D=\ell_{D B_{2}}^{D_{1}} \cap O I \Longrightarrow\left\{\begin{array}{c}
I B_{2} \| B D_{1} \\
D B_{2} \|[B * D] D_{1} .
\end{array}\right\}
$$

Choose the point $B_{1}$ (Fig.12) as auxiliary points for the construction of multiplication $A *(B * D)$. Construct the line $\ell_{(B * D) B 1}^{P_{1}}$. Then, according to (2) have: 
1. $B_{1} \notin O I$,

2. $O B_{2} \cap \ell_{I B_{1}}^{A}=P_{1}, \quad \Longrightarrow A *(B * D)=\ell_{(B * D) B_{1}}^{P_{1}} \cap O I$

3. $\ell_{(B * D) B_{1}}^{P_{1}} \cap O I=C$.

$$
\Longrightarrow\left\{\begin{array}{c}
I B_{1} \| A P_{1} \\
(B * D) B_{1} \|[A *(B * D)] P_{1}
\end{array}\right\}
$$

Whereas, the 3.4 and (3.5), have

$$
\left\{\begin{array}{c}
B D_{1} \|(A * B) P_{2} \\
(B * D) D_{1} \|[(A * B) * D] P_{2}
\end{array}\right\}
$$

We examine three-vertices $B B_{1} D_{1}$ and $(A * B) P_{1} P_{2}$, to which, by (3.3) have $B B_{1} \|(A * B) P_{1}$ and from (3.7) we have $B D_{1} \|(A * B) P_{2}$. Therefore, the Desargues affine plane theorem, have $B_{1} D_{1} \| P_{1} P_{2}$. We examine further three-vertices $(B * D) B_{1} D_{1}$ and $[(A * B) * D] P_{1} P_{2}$, for which, from above we have $B_{1} D_{1} \| P_{1} P_{2}$ and from (3.7) we have $(B * D) D_{1} \|[(A * B) * D] P_{2}$. Therefore we take from $(B * D) B_{1} \|[(A * B) * D] P_{1}$. But by (3.6) we have also $(B * D) B_{1} \|[A *(B * D)] P_{1}$, that brings $[(A * B) * D] P_{1} \|$ $[A *(B * D)] P_{1}$, and since the points $(A * B) * D, A *(B * D) \in O I$, we take $(A * B) * D=A *(B * D)$.

Proposition 3.3. For every point except $O$ in $O I$, there exists its right symmetrical according to multiplication:

$$
(\forall A \in O I-\{O\})\left(\exists A^{-1} \in O I-\{O\}\right) A * A^{-1}=I
$$

Proof. We distinguish two cases: $A=I$ and $A \neq I, O$.

Case 1. If $A=I$, then $A^{-1}=I$ because, according to 3.1$), I * I=I$.

Case 2. If $A \neq I, O$, requested points $A^{-1} \in O I$, such that

$$
\begin{aligned}
& \text { 1. } A_{1}^{-1} \notin O I, \\
& \text { 2. } \ell_{I A_{1}^{-1}}^{A} \cap O A_{1}^{-1}=P_{1}, \\
& \text { 3. } \ell_{A^{-1} A_{1}^{-1}}^{P_{1}} \cap O I=I .
\end{aligned}
$$

Given this, we take initially a point $A_{1}^{-1} \notin O I$ and construct the line $I A_{1}^{-1}$, and then the line $\ell_{I A_{1}^{-1}}^{A}$. Mark $P_{1}=\ell_{I A_{1}^{-1}}^{A} \cap O A_{1}^{-1}$. Furthermore construct the line $I P_{1}$ and parallel with it by the points $A_{1}^{-1}$ construct the line $\ell_{I P_{1}}^{A_{1}^{-1}}$. The latter is not parallel with the line $O I$, therefore expects that at some point: $\ell_{I P_{1}}^{A_{1}^{-1}} \cap O I \neq O$. It is clear that this point is the point $A^{-1}$ 


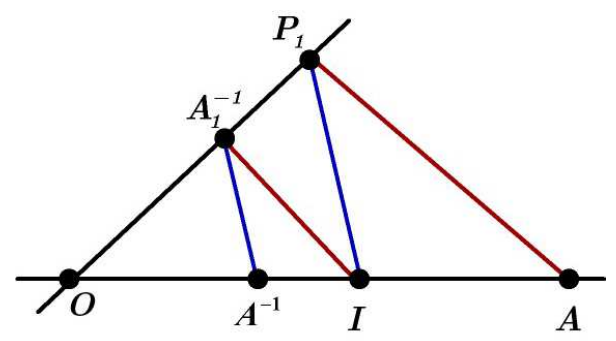

Figure 13.

(Fig.13), such that the $A * A^{-1}=I$ and $A^{-1} \neq O$. So the $A^{-1}$ thus the resulting the right identity element of point $A$.

Due to the definition of group, 4, from Propositions 3.1, 3.2 and 3.3 we obtain this

Theorem 4. In an Desargues affine plane the groupoid $(O I, *)$ is a group; identity element is the point $I$.

Based on one theorem of algebra, right neutral element of an element of one group is neutral element of that element, [4], 5]. Therefore,

$$
(\forall A \in O I-\{O\})\left(\exists A^{-1} \in O I-\{O\}\right) A * A^{-1}=A^{-1} * A=I
$$

4. The algebra $(O I,+, *)$ is a Corp in Desargues affine plane

Proposition 4.1. The multiplication $*$ is distributive related to the addition + in the line $O I$ :

$$
\begin{aligned}
& \text { (i) }(A+B) * D=A * D+B * D \\
& \text { (ii) } A *(B+D)=A * B+A * D
\end{aligned}
$$

for every $A, B, D \in O I$.

Proof. (i) In the case where at least one of the points A, B, D is the point $\mathrm{O}$, by the (2.3), equivalence $(i)$ is evident. We eliminate in the case where $A, B, D \neq O$ and $A \neq B \neq D$ (when at least two points are the same, equally justify). We distinguish two sub-cases: $a$ ) at least one of the points $A, B, D$ is the point $I ; b) A, B, D \neq I$.

a) When $D=I$, according to (3.1), equalization $(i)$ is evident. Let it be now $A=I$ (the case $B=I$ behaves in case $A=I$, based on a 


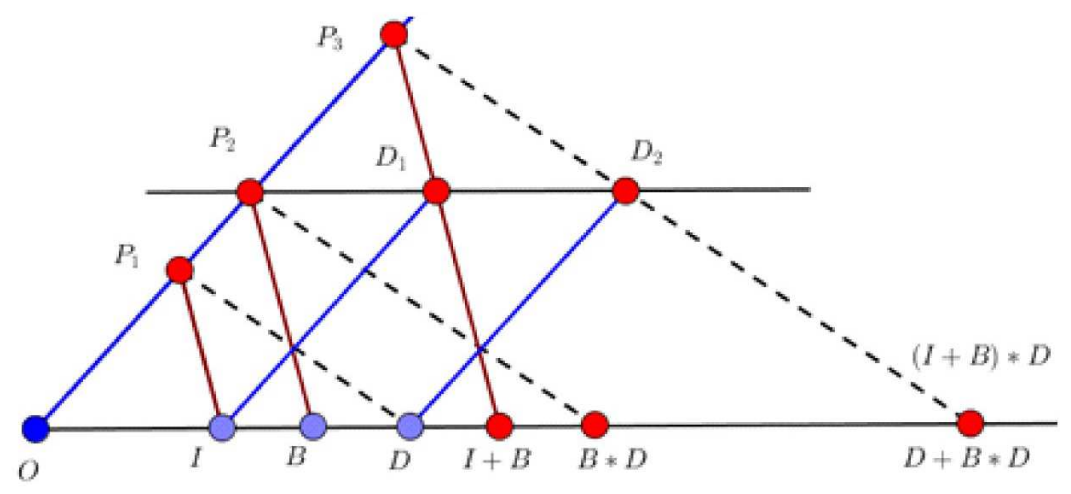

Figure 14.

commutative addition property in $O I$ ). For as much as $A \neq B \neq D$, have $B \neq I$ and $D \neq I$ (Fig.14). Equalisation $(i)$, in this case takes the view $(I+B) * D=D+B * D$.

For the construction of multiplication $(I+B) * D$, construct firstly multiplication $B * D$, taking its auxiliary point the point $P_{1} \notin O I$. Mark $P_{2}=O P_{1} \cap \ell_{I P_{1}}^{B}$. Then, according to the algorithm Algorithm 2, where in the role of $A$ is $B$, in the role of $B$ is $D$, in the role of $B_{1}$ is $P_{1}$, in the role of $P_{1}$ is $P_{2}$, have

$$
\begin{aligned}
& \left\{\begin{array}{l}
\text { 1. } P_{1} \notin O I, \\
\text { 2. } O P_{1} \cap \ell_{I P_{1}}^{B}=P_{2}, \\
\text { 3. } \ell_{D P_{1}}^{P_{2}} \cap O I=C .
\end{array}\right\} \Longrightarrow B * D=\ell_{D P_{1}}^{P_{2}} \cap O I \\
& \Longrightarrow\left\{\begin{array}{c}
I P_{1} \| B P_{2} \\
D P_{1} \|(B * D) P_{2}
\end{array}\right\}
\end{aligned}
$$

Construct further the sum $I+B$, by taking its auxiliaries point the point $P_{2} \notin O I$. Mark $D_{1}=\ell_{O I}^{P_{2}} \cap \ell_{O P_{2}}^{I}$. Then, according to the Algorithm 1, where in the role of $A$ is $I$, in the role of $B_{1}$ is $P_{1}$, in the role of $P_{1}$ is $P_{2}$, have

$$
\begin{gathered}
\left\{\begin{array}{l}
\text { 1. } P_{2} \notin O I, \\
\text { 2. } \ell_{O I}^{P_{2}} \cap \ell_{O P_{2}}^{I}=D_{1}, \\
\text { 3. } \ell_{B P_{2}}^{D_{1}} \cap O I=C .
\end{array}\right\} \Longrightarrow I+B=\ell_{B P_{2}}^{D_{1}} \cap O I \\
\quad \Longrightarrow\left\{\begin{array}{c}
O P_{2} \| I D_{1} \\
B P_{2} \|(I+B) D_{1} .
\end{array}\right\}
\end{gathered}
$$


Finally construct multiplication $(I+B) * D$, by taking its auxiliaries point the point $P_{1} \notin O I$. Mark $P_{3}=O P_{1} \cap \ell_{I P_{1}}^{(I+B)}$. Then, according to the Algorithm 2, where in the role of $A$ is $I+B$, in the role of $B$ is $D$, in the role of $B_{1}$ is $P_{1}$, in the role of $P_{1}$ is $P_{3}$, have

$$
\begin{aligned}
& \text { 1. } P_{1} \notin O I, \\
& \text { 2. } O P_{1} \cap \ell_{I P_{1}}^{(I+B)}=P_{3}, \Longrightarrow(I+B) * D=\ell_{D P_{1}}^{P_{3}} \cap O I \\
& \text { 3. } \ell_{D P_{1}}^{P_{3}} \cap O I=C . \\
& \qquad\left\{\begin{array}{c}
I P_{1} \|(I+B) P_{3} \\
D P_{1} \|[(I+B) * D] P_{3} .
\end{array}\right\}
\end{aligned}
$$

Now construct the right side $D+B * D$ of equivalence, by taking as the auxiliaries point of sum the point $P_{2} \notin O I$. Mark $D_{2}=\ell_{O I}^{P_{2}} \cap \ell_{O P_{2}}^{D}$. Then, according to the Algorithm 1, where in the role of $A$ is $D$, in the role of $B$ is $B * D$, in the role of $B_{1}$ is $P_{2}$, in the role of $P_{1}$ is $D_{2}$, have:

$$
\begin{aligned}
& \text { 1. } P_{2} \notin O I, \\
& \text { 2. } \ell_{O I}^{P_{2}} \cap \ell_{O P_{2}}^{D}=D_{2}, \quad \Longrightarrow D+(B * D)=\ell_{(B * D) P_{2}}^{D_{2}} \cap O I \\
& \text { 3. } \ell_{(B * D) P_{2}}^{D_{2}} \cap O I=C . \\
& \qquad \Longrightarrow\left\{\begin{array}{cc}
O P_{2} \| D D_{2} \\
(B * D) P_{2} \|[D+(B * D)] D_{2}
\end{array}\right\}
\end{aligned}
$$

By (4.2), (4.3) and (4.4) we have that $I P_{1}\left\|B P_{2}\right\|(I+B) D_{1} \|(I+B) P_{3}$, which indicates that the points $(I+B), D_{1}$ and $P_{3}$ are collinear.

We note that three-vertices $I D P_{1}$ and $D_{1} D_{2} P_{3}$ have respective vertices in parallel lines $I D_{1}\left\|P_{1} P_{2}\right\| D D_{2}$ and satisfy the Desargues affine plane theorem (DAPT) conditions, therefore

$$
\begin{aligned}
& I P_{1}\left\|D_{1} P_{3} \stackrel{(D A P T)}{\Longrightarrow} I D_{1}\right\| D_{2} P_{3} .
\end{aligned}
$$

But, by (4.2) and (4.5), we have $D P_{1} \|[D+B * D] D_{2}$. Since the parallelism is equivalence relation (Proposition 1.1), by the (4.6), we have $D_{2} P_{3} \|$ $[D+(B * D)] D_{2}$. So the, points $P_{3}, D_{2}$ and $[D+(B * D]$ are collineary. By (4.4) have $D P_{1} \|[(I+B) * D] P_{3}$, that brings $[D+(B * D)] P_{3} \|[(I+B) * D] P_{3}$. Consequently the resulting true equalization

$$
(I+B) * D=D+B * D
$$

b) $A, B, D \neq I$, where $A \neq B \neq D$ 


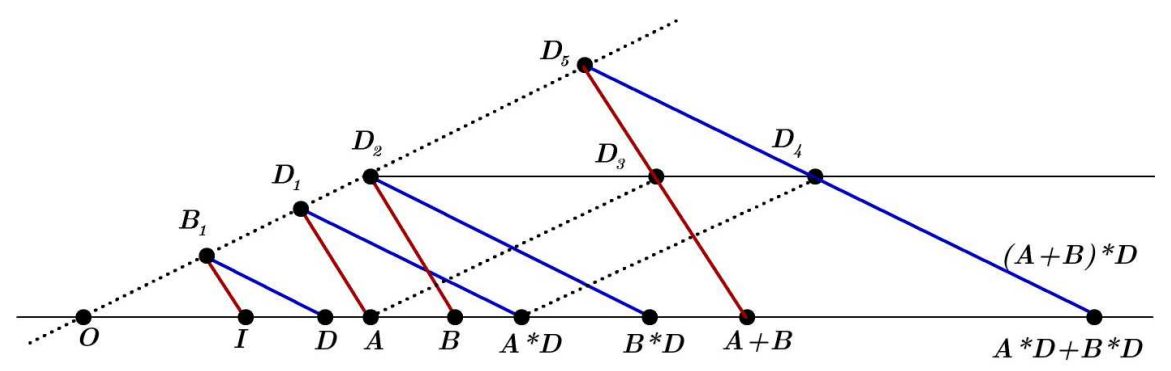

Figure 15.

To the construction of multiplication $(A+B) * D$, initially construct $A * D$ and $B * D$, (Fig.15). To multiplication $A * D$, according to Algorithm 2, where in the role of $B$ is $D$, in the role of $P_{1}$ is $D_{1}$, have

1. $B_{1} \notin O I$,

2. $O B_{1} \cap \ell_{I B_{1}}^{A}=D_{1}, \quad \Longrightarrow A * D=\ell_{D B_{1}}^{D_{1}} \cap O I$.

3. $\ell_{D B_{1}}^{D_{1}} \cap O I=C$.

Whereas for production $B * D$ have

1. $B_{1} \notin O I$,

2. $O B_{1} \cap \ell_{I B_{1}}^{B}=D_{2}, \quad \Longrightarrow B * D=\ell_{D B_{1}}^{D_{2}} \cap O I$.

3. $\ell_{D B_{1}}^{D_{2}} \cap O I=C$.

From 4.7) and (4.8), have

$$
\begin{gathered}
I B_{1}\left\|A D_{1}\right\| B D_{2} \\
D B_{1}\left\|(A * D) D_{1}\right\|(B * D) D_{2}
\end{gathered}
$$

Construct further the sum $A+B$, by taking as its auxiliaries point, the point $D_{2} \in O B_{1}$. Mark $D_{3}=\ell_{O I}^{D_{2}} \cap \ell_{O D_{2}}^{A}$. Then, according to the Algorithm 1 , where in the role of $B_{1}$ is $D_{2}$, in the role of $P_{1}$ is $D_{3}$, have

$$
\begin{gathered}
\left\{\begin{array}{l}
\text { 1. } D_{2} \notin O I, \\
\text { 2. } \ell_{O I}^{D_{2}} \cap \ell_{O D_{2}}^{A}=D_{3}, \\
\text { 3. } \ell_{D_{2} B}^{D_{3}} \cap O I=C .
\end{array}\right\} \Longrightarrow A+B=\ell_{D_{2} B}^{D_{3}} \cap O I \\
\Longrightarrow\left\{\begin{array}{c}
D_{2} D_{3} \| O I \\
A D_{3} \| O D_{2} \\
B D_{2} \|(A+B) D_{3}
\end{array}\right\}
\end{gathered}
$$


CORP IN THE SET OF POINTS IN A LINE OF DESARGUES AFFINE PLANE 43

Finally construct production $(A+B) * D$, by taking as its auxiliaries point, the point $B_{1} \notin O I$. Mark $D_{5}=O B_{1} \cap \ell_{I B_{1}}^{(A+B)}$. Then, according to the Algorithm 2, where in the role of $A$ is $A+B$, in the role of $B$ is $D$, in the role of $P_{1}$ is $D_{5}$, have

\section{1. $B_{1} \notin O I$,}

2. $O B_{1} \cap \ell_{I B_{1}}^{(A+B)}=D_{5}, \quad \Longrightarrow(A+B) * D=\ell_{D B_{1}}^{D_{5}} \cap O I$

3. $\ell_{D B_{1}}^{D_{5}} \cap O I=C$.

$$
\Longrightarrow\left\{\begin{array}{c}
I B_{1} \|(A+B) D_{5} \\
D B_{1} \|[(A+B) * D] D_{5}
\end{array}\right\}
$$

Now construct the right-hand of the equalization (i) $A * D+B * D$, by taking as auxiliaries point of the sum the point $D_{2} \notin O I$. Mark $D_{4}=$ $\ell_{O I}^{D_{2}} \cap \ell_{O D_{2}}^{A * D}$. Then, according to the Algorithm 1, where in the role of $A$ is $A * D$, in the role of $B$ is $B * D$, in the role of $B_{1}$ is $D_{2}$, in the role of $P_{1}$ is $D_{4}$, have

$$
\begin{aligned}
& \left\{\begin{array}{l}
\text { 1. } D_{2} \notin O I, \\
\text { 2. } \ell_{O I}^{D_{2}} \cap \ell_{O D_{2}}^{A * D}=D_{4}, \\
\text { 3. } \ell_{D_{2}(B * D)}^{D_{4}} \cap O I=C .
\end{array}\right\} \Longrightarrow A * B+B * D=\ell_{D_{2}(B * D)}^{D_{4}} \cap O I \\
& \quad \Longrightarrow\left\{\begin{array}{c}
O D_{2} \|(A * D) D_{4} \\
(B * D) D_{2} \|[(A * B)+(B * D)] D_{4}
\end{array}\right\}
\end{aligned}
$$

From 4.9, 4.10 and 4.11) we have

$$
I B_{1}\left\|A D_{1}\right\| B D_{2}\left\|(A+B) D_{5}\right\|(A+B) D_{3}
$$

which indicates that the points $A+B, D_{3}, D_{5}$, are collinear points. Consequently $D_{3} \in(A+B) D_{5}$, that brings

$$
A D_{1} \| D_{3} D_{5}
$$

Also, from the 4.9, 4.11) and 4.12, it turns out that

$$
\begin{gathered}
D_{1} B_{1}\left\|(A * D) D_{1}\right\|(B * D) D_{2}\left\|[(A+B) * D] D_{5}\right\| \\
\|[(A * D)+(B * D)] D_{4}
\end{gathered}
$$

We note that three-vertices $A(A * D) D_{1}$ and $D_{3} D_{4} D_{5}$ meet the conditions of the Desargues axiom (D1) (Proposition 1.3), since, from the (4.10) and 4.12, we have that:

$$
D_{1} D_{5}\left\|A D_{3}\right\|(A * D) D_{4}
$$


Therefore from 4.10 and 4.14 we have

$$
\begin{gathered}
A(A * D) \| D_{3} D_{4} \\
A D_{1} \| D_{3} D_{5}
\end{gathered} \stackrel{\mathrm{D} 1}{\Longrightarrow}(A * D) D_{1} \| D_{4} D_{5}
$$

Whereas, from 4.15) and 4.17) the resulting that also points $(A+B) *$ $D, D_{4}, D_{5}$ are collinear points. Consequently $D_{4} \in[(A+B) * D] D_{5} \|$ $D B_{1}$, that implies

$$
[(A+B) * D] D_{5} \|[(A * D)+(B * D)] D_{4} .
$$

Namely

$$
(A+B) * D=(A * D)+(B * D)
$$

(ii) The proof of equation $(i i)$ is analog. However, we present another proof, accepting that, is in similar way with the proof in point a) of equation $(I+B) * D=D+B * D$, it is also a proof of the equation

$$
A *(I+D)=A+A * D
$$

in the case where $A, B, D \neq O$ and $A \neq B \neq D$. In this case, since $B \neq O$, from 3.8 , exists the point $B^{-1}$. Then:

$$
\begin{aligned}
{[A *(B+D)] * B^{-1} } & \stackrel{(6)}{=} A *\left[(B+D) * B^{-1}\right] \\
& \stackrel{(4.1 . i)}{=} A *\left(B * B^{-1}+D * B^{-1}\right) \\
& \stackrel{(3.8)}{=} A *\left(I+D * B^{-1}\right) \\
& \stackrel{(4.18)}{=} A+A *\left(D * B^{-1}\right) \\
& \stackrel{(3.1),(3.3)}{=} A * I+(A * D) * B^{-1} \\
& \stackrel{(3.8)}{=} A *\left(B * B^{-1}\right)+(A * D) * B^{-1} \\
& \stackrel{(3.2)}{=}(A * B) * B^{-1}+(A * D) * B^{-1} \\
& \stackrel{(4.1 . i)}{=}[(A * B)+(A * D)] * B^{-1}
\end{aligned}
$$

From here we have

$$
\begin{aligned}
{[A *(B+D)] * B^{-1} } & =[(A * B)+(A * D)] * B^{-1} \Longrightarrow \\
A *(B+D) & =(A * B)+(A * D) .
\end{aligned}
$$


CORP IN THE SET OF POINTS IN A LINE OF DESARGUES AFFINE PLANE 45

Bearing in mind the Theorem 2 and the Propositions 3.1, 3.2 and 4.1 we obtain this

Theorem 5. In Desargues affine plane the algebra $(O I,+, *)$ is the unitary ring.

Theorem 6. In Desargues affine plane the algebra $(O I,+, *)$ is a corp (skew field).

Proof. Since $I \neq O$, in the ring $O I$ has at least one non-zero element. Then, by definition of the a skew-fields (see [5, 19, 20, 21]) requested to prove as follows:

1. $O I^{*}=O I-\{O\}$, is stable subset of $O I$ about multiplication. To really, if the points $A, B \in O I^{*}$, then also $A * B \in O I^{*}$. We suppose $A * B=O$. For as much as $A \neq O$, by (3.2) and (3.8) we have $B=I * B=$ $\left(A^{-1} * A\right) * B=A^{-1} *(A * B)=A^{-1} * O=O$.

This contradicts the condition that $B \neq O$.

2. The groupoid $\left(O I^{*}, *\right)$ is a group, because it is a subgroup of $(O I, *)$ which, according to Theorem 4 , is a group.

\section{REFERENCES}

[1] O. Zaka, K. Filipi, The transform of a line of desargues affine plane in an additive group of its points, International Journal of Current Research, 8(07) (2016), 3498334990. https://arxiv.org/abs/1609.01155

[2] O. Zaka, K. Filipi, One construction of an affine plane over a corps, Journal of advances in mathematics, Council for Innovative Research, 12(5) (2016), 62006206.

[3] O. Zaka, K. Filipi, An Application of Finite Affine Plane of Order n, in an Experiment Planning, International Journal of Science and Research (IJSR), 6(6) (2017), 1744-1747.

[4] K. Filipi, Abstract Algebra, First Edition, Mirgeralb Publishing House, Tirana, Albania, 2013 (in Albanian).

[5] K. Filipi, Algebra and Geometry, Republished, Edlora Publishing House, Tirana, Albania, 2015 (in Albanian).

[6] F. Borceux, An Axiomatic Approach to Geometry (Geometric Trilogy I), Springer International Publishing Switzerland, 2014.

[7] F. Borceux, An Algebraic Approach to Geometry(Geometric Trilogy II), Springer International Publishing Switzerland, 2014.

[8] M. Berger, Geometry I, Springer-Verlag Berlin Heidelberg, 1987.

[9] O. Zaka, Contribution to reports of some algebraic structures with affine plane geometry and applications, Ph.D. thesis, Polytechnic University of Tirana, Tirana, Albania, Department of Mathematical Engineering, 2016 (in Albanian).

[10] F. Sadiki, Models of Ternary Algebraic Structures in Projective Geometry, Ph.D. thesis, Polytechnic University of Tirana,Tirana, Albania, Department of Mathematical Engineering, 2015 (in Albanian). 
[11] D. R. Hughes, F. C. Piper, Projective Planes, Springer-Verlag New York Inc. and Springer-Verlag New York Heidelberg Berlin, 1973.

[12] J. D. Dixon, B. Mortimer, Permutation Groups, Graduate Texts in Mathematics, Vol 163, Springer-Verlag New York Berlin Heidelberg, 1996.

[13] P. A. Grillet, Abstract Algebra, Graduate Texts in Mathematics (Second Edition), Springer Science + Business Media, LLC, 2007.

[14] S. Lang, Algebra, Graduate Text in Mathematics, (Vol. 211) (Third Edition), Springer-Verlag New York, 2002.

[15] J. J. Rotman, An Introduction to the Theory of Groups, Graduate text in mathematics, 4th edition, Springer Verlag, 1995.

[16] O. A. Gallian, Contemporary Abstract Algebra Broks/Cole (Eighth Edition), Cengage Larning, 2013.

[17] F. Rothe, Several topics from geometry. Topics from Geometry, 2010. http://math2.uncc.edu/ frothe/

[18] R. Hartshorne, Geometry: Euclid and Beyond, Springer-Verlag New York Berlin Heidelberg, 2000.

[19] A. H. Schofield, Representation of rings over skew fields, London Mathematical Society Lecture Note Series: 92. Cambridge University Press, 1985.

[20] K. Mathiak, Valuations of Skew Fields and Projective Hjelmslev Spaces, Lecture Notes in Mathematics, Springer-Verlag Berlin Heidelberg New York Tokyo, 1986.

[21] E. Kleinert, Units in Skew Fields, Springer Basel AG. Originally published by Birkhăuser Verlag, Basel, 2000.

[22] S. Bilaniuk, A problem course on projective planes, Trent University, Ontario, Canada, 2003.

Kristaq Filipi

Polytechnic University of Tirana,

Faculty of Mathematical and Physical Engineering,

Department of Mathematical Engineering,

Street Sulejman Delvina, Tirana, Republic of Albania

E-mail address: f_kristaq@hotmail.com

ORgest ZAKA

University of Vlora 'Ismail Qemali', Vlora,

Faculty of Technical Science,

Department of Mathematics,

St. Kosovo, Neighborhood Independence, 9400, Skela, Vlora,

Republic of Albania

E-mail address: orgest.zaka@univlora.edu.al

AzIR Jusufi

University of Tetova, Tetovo, Macedonia

Faculty of Natural Sciences and Mathematics

Department of Mathematics

Str. Ilinden, Nn.1200 Tetova,

Republic of North Macedonia

E-mail address: azir.jusufi@unite.edu.mk 